\title{
Heterogeneous Expectations, Indeterminacy, and Postwar US Business Cycles
}

\author{
Francisco ILABACA \\ University of California, Irvine
}

\author{
Fabio MILANI* \\ University of California, Irvine
}

March, 2020

\begin{abstract}
This paper estimates a New Keynesian model extended to include heterogeneous expectations, to revisit the evidence that postwar US macroeconomic data can be explained as the outcome of passive monetary policy, indeterminacy, and sunspot-driven fluctuations in the pre1979 sample, with a switch to active monetary policy and a determinate equilibrium starting in the early 1980s. Different shares of consumers and firms form either rational expectations, or adaptive and extrapolative expectations. The inclusion of heterogeneous expectations alters the determinacy properties of the model compared to the corresponding case under exclusively rational expectations. The Taylor principle is neither necessary nor sufficient, as the details of expectations may matter more for equilibrium stability.

The model is estimated with Bayesian techniques, using rolling windows and allowing the parameters to fall both in the determinacy and indeterminacy regions. The estimates reveal large shares of agents who depart from rational expectations; heterogeneous expectations are preferred by the data everywhere in the sample. The results confirm that macroeconomic data in the early windows are better explained by indeterminacy, while determinacy is favored over the latest two decades. We uncover, however, some subsamples that include the 1980s and 1990s in which the Taylor principle is satisfied, but expectations becoming extrapolative raise the probability of indeterminacy to $50 \%$ and more.
\end{abstract}

Keywords: Heterogeneous Expectations in New Keynesian Model, Indeterminacy, Sunspots, Taylor Principle, Deviations from Rational Expectations, Time-Varying Parameters.

JEL classification: E32, E52, E58, E70.

\footnotetext{
*Address for Correspondence: 3151 Social Science Plaza, Department of Economics, University of California, Irvine, CA 92697-5100.
} 


\section{Introduction}

A generally accepted account of US postwar macroeconomic history views output and inflation fluctuations in the 1960-1970s as the outcome of passive monetary policy and indeterminacy, followed by a shift to active monetary policy after 1979, which induced stability in the macroeconomic environment by ensuring a determinate equilibrium.

Empirical evidence in favor of this narrative is provided, for example, in the influential papers by Clarida, Galí, and Gertler (2000), and Lubik and Schorfheide (2004). Their works, as well as the related literature, use New Keynesian frameworks that particularly highlight the role of monetary policy in managing private-sector expectations. A weak monetary policy response may cause inflation and output expectations to become unhinged, steering the economy away from the rational expectations equilibrium. Expectations may coordinate, instead, around a more volatile equilibrium, where realizations of a sunspot variable affect agents' beliefs and, ultimately, the dynamics of macroeconomic variables.

The results on the transition from indeterminacy to determinacy are, however, obtained in models that impose two strong expectational assumptions: first, that expectations are formed according to the rational expectations hypothesis, and, second, that expectations are homogeneous. All households and firms in the model are assumed to form identical expectations conditioned on the same, correct, model of the economy.

But recent studies have provided growing evidence in favor of heterogeneous expectations. Branch (2004) uses individual-level data on inflation expectations from the Michigan Survey of Consumers to show that different shares of consumers form expectations based on alternative forecasting models, with different levels of complexity. Mankiw, Reis, and Wolfers (2004) and Branch (2014) document a significant extent of disagreement among professional forecasters. The experimental evidence has also strongly stressed the importance of heterogeneous expectations: Hommes (2011, 2013) show that expectations cluster around different groups, with some characterized by adaptive, and others by trend-following, behavior.

Branch and McGough (2009) introduce heterogeneous expectations in a New Keynesian model and analyze their microfoundations. The model retains a share of agents who form conventional rational expectations, but they allow for a share of agents whose expectations deviate from rational (RE) and can be adaptive, naïve, or extrapolative. As they stress, the inclusion of heterogeneity 
alters the determinacy properties of the system, and may be stabilizing or destabilizing depending on the nature of the expectations. If expectations have an adaptive component, that is they respond less than one-to-one with respect to the lagged value of the endogenous variable, a larger share of non-RE agents will be stabilizing. The determinacy region is expanded, and the equilibrium can be determinate even with weak responses to inflation. On the other hand, if expectations are extrapolative, by responding more than one-to-one to past values, indeterminacy will be more likely. For large enough shares of non-RE agents, conventional active monetary policies become powerless in trying to steer the economy toward a stable equilibrium. Hence, the Taylor principle is neither necessary nor sufficient for equilibrium uniqueness. As a consequence, it is possible that determinacy exists in the 1970s in conjunction with passive monetary policy, or that indeterminacy continues to provide a better explanation even of the post-Volcker data, as long as beliefs are extrapolative. The details of expectations, overall, may be more important than monetary policy for determinacy of the equilibrium.

In this paper, we estimate the New Keynesian model with heterogeneous expectations and allow for both determinacy and indeterminacy. We use the techniques developed in Bianchi and Nicoló (2019) to solve the model and obtain the likelihood under indeterminacy. We also recognize that monetary policy coefficients and the formation of expectations may have changed over time. Therefore, we adopt a rolling-window estimation from 1954 to the end of the sample. We use different window sizes: in the benchmark estimation, our rolling window includes twenty years of observations, but we also repeat the estimation with a ten-year window. The model is re-estimated every year, and we calculate how the probability of determinacy versus indeterminacy varies over time. We use a rolling sample because, while there may be evidence of a structural break for monetary policy coefficients in correspondence of the appointment of Paul Volcker as the Federal Reserve's Chairman in 1979, we cannot realistically assume a one-time structural break in the expectation parameters, or in other structural coefficients.

Results. The estimation results strongly indicate that heterogeneous expectations provide a better fit of the data than homogeneous expectations do, as measured by the marginal likelihoods. We estimate large shares of non-RE agents: at points in the sample, they reach up to $80 \%$ for output expectations and more than $60 \%$ for inflation expectations.

We document ample evidence of time-variation in the structural and expectation parameters. Monetary policy is passive toward inflation in the earlier samples, as expected, and it becomes 
more reactive to the output gap for windows that are centered around the 1970s. The shares of non-RE agents vary over time. They are low in the earlier windows and for some of the samples that begin in the second half of the 1970s, but, in general, they indicate that the vast majority of agents do not conform with rational expectations. We also document that expectations about the output gap display backward-looking coefficients close to one over most of the sample. In contrast, inflation expectations are typically formed adaptively, with coefficients ranging from below 0.5 to 0.7, but with the exception of windows that start in the late 1970s, when the coefficients rise above one and expectations display extrapolative or trend-chasing behavior.

Parts of the conventional story on indeterminacy is confirmed. The early years in our sample are best explained as coming from a data-generating process characterized by passive monetary policy and multiple equilibria. For samples starting after the early 1980s, there's a definite shift to determinacy. But there are other periods in which the Taylor principle is usually considered satisfied in the literature, but where we find expectations that become extrapolative. In these periods, the probability of indeterminacy rises considerably, up to $70 \%$. These additional episodes of potential indeterminacy are usually absent in estimations under homogeneous expectations. Under indeterminacy, the economy is susceptible to the action of sunspots. Based on our estimates, sunspot shocks typically don't explain a large share of fluctuations in output and inflation, but they can account for up to $20 \%$ in some episodes. Sunspots also account for a similar share of the variability in inflation in those samples where expectations become extrapolative.

Related Literature. This paper aims to contribute to multiple literatures. More directly, it builds empirical evidence in favor of modeling heterogeneous expectations in the New Keynesian model. The microfoundations of heterogeneous expectations were developed in Branch and McGough (2009), who also document the conditions for equilibrium determinacy and show that heterogeneity can expand or shrink the region of determinacy depending on the nature of non-RE expectations. ${ }^{1}$ Beqiraj et al. (2018) estimate shares of agents that depart from rational expectations ranging from $13 \%$ to $46 \%$; they don't consider the implications of heterogeneous expectations for indeterminacy in the estimation, and don't allow for time variation in the parameters. Our empirical estimates suggest that non-RE shares are even larger. Other papers have studied the implications of heterogeneous expectations on the amplification of technology shocks on output

\footnotetext{
${ }^{1}$ Massaro (2013) adds heterogeneous expectations in the microfoundations of a New Keynesian model with longhorizon expectations instead.
} 
(Branch and McGough, 2011), optimal monetary policy (Gasteiger, 2014, Di Bartolomeo et al., 2017, Beqiraj et al., 2019), and monetary-fiscal policy interactions (Gasteiger, 2018). While we provide evidence on the importance of expectation heterogeneity at the macro level, other papers have documented its importance on micro data (Branch, 2004, 2014, Pesaran and Weale, 2006, Dovern et al., 2012, Cole and Milani, 2020), or in laboratory experiments (Hommes, 2011, 2013). Cole and Milani (2019) use a DSGE-VAR approach and reveal that a major misspecification in the New Keynesian model lies in the way expectations are modeled: when heterogeneous expectations, chosen to mirror lab evidence, replace homogeneous rational expectations, the data become more supportive of DSGE restrictions. Calvert Jump and Levine (2019) show that the model with heterogeneous expectations can account for some empirical regularities (excess kurtosis, stochastic volatility, and departures from rational expectations) that cannot be explained by the benchmark New Keynesian model.

The paper then adds to the literature on changes in monetary policy and indeterminacy to explain postwar US economic history. The paper generally confirms the shift from indeterminacy to determinacy over the sample found in Clarida, Galí, and Gertler (2000) and Lubik and Schorfheide (2004), but it uncovers additional periods when indeterminacy may arise because of extrapolative expectations. Other papers relax rational expectations or add behavioral elements to revisit the empirical evidence in favor of indeterminacy, but they impose homogeneous expectations (Milani, 2008, Ilabaca, Meggiorini, and Milani, 2020).

Benhabib and Farmer (1999) and Farmer (2019) review the broader literature on indeterminacy in macroeconomics. Empirical studies have been few in the past due to difficulties in solving and estimating models with multiple equilibria. Lubik and Schorfheide (2004), Farmer, Khramov, and Nicoló (2015), and Bianchi and Nicoló (2019) provide key methodological contributions that make it easier to take these models to the data. Our paper adds to the literature by revisiting the empirical evidence for indeterminacy versus determinacy in a model that studies the interaction between monetary policy and expectations, by allowing for heterogeneous expectations and parameter variation.

Furthermore, the paper is connected to the literature that deals with parameter instability in DSGE models. Fernandez-Villaverde and Rubio-Ramirez (2008) estimate a model with timevarying parameters to judge whether parameters can be interpreted as 'structural'. Canova (2009), Canova and Ferroni (2012), and Castelnuovo (2012), use a closer approach to ours, by employing a 
rolling window estimation. Compared to models with Markov-Switching, time-varying parameters, or stochastic volatilities, a rolling Bayesian estimation allows us to consider time variation in all parameters at the same time in a computationally tractable way (the other approaches typically only allow a minor subset of parameters to shift, while forcing others to remain constant). These papers still impose determinacy and work with models with homogeneous expectations, while we emphasize parameter variation related to heterogeneous expectations, and we show how parameters may fall in regions that are conducive to indeterminacy.

Finally, the paper is more broadly connected to the literatures that model deviations from rational expectations, such as the literature on adaptive learning in macroeconomics (e.g., Evans and Honkapohja, 2001). We don't directly model agents' learning here, but we show how the parameters related to expectations change over time through our rolling window estimation.

\section{New Keynesian Model with Heterogeneous Expectations}

We assume that the evolution of the economy can be summarized by the following New Keynesian model, extended to allow for heterogeneous expectations, as in Branch and McGough (2009, 2018):

$$
\begin{aligned}
& x_{t}=\tilde{E}_{t} x_{t+1}-\sigma^{-1}\left(i_{t}-\tilde{E}_{t} \pi_{t+1}\right)+g_{t} \\
& \pi_{t}=\beta \tilde{E}_{t} \pi_{t+1}+\kappa x_{t}+u_{t} \\
& i_{t}=\rho_{i} i_{t-1}+\left(1-\rho_{i}\right)\left(\psi_{\pi} \pi_{t}+\psi_{x} x_{t}\right)+\varepsilon_{t} \\
& g_{t}=\rho_{g} g_{t-1}+\varepsilon_{t}^{g} \\
& u_{t}=\rho_{u} u_{t-1}+\varepsilon_{t}^{u},
\end{aligned}
$$

where $x_{t}$ denotes the output gap, $\pi_{t}$ denotes inflation, and $i_{t}$ denotes the short-term nominal interest rate, which serves as the monetary policy instrument. All variables are in log deviations from steady-state. The parameter $\sigma$ denotes the intertemporal elasticity of substitution, $\beta$ the household's discount factor, and $\kappa \equiv \frac{\left(1-\xi_{p} \beta\right)(1-\beta)}{\xi_{p}} \frac{\omega+\sigma}{1+\omega \epsilon}$ is a composite parameter that captures the slope of the Phillips curve; $\kappa$ is an inverse function of the degree of price stickiness (the Calvo parameter $\xi_{p}$ ), and it depends on the elasticity of marginal costs to income (denoted by $\omega$ ), and on the steady-state markup of prices over marginal costs $(\epsilon)$.

Equation (1) is the Euler equation, which expresses the output gap as a function of one-periodahead expectations, and of the ex-ante real interest rate; $g_{t}$ denotes a demand disturbance (for 
example, due to exogenous shifts in consumer tastes or government spending). Equation (2) is a New Keynesian Phillips curve: inflation depends on expected inflation, on the current output gap, and on the exogenous cost-push disturbance $u_{t}$. Both $g_{t}$ and $u_{t}$ evolve as $\operatorname{AR}(1)$ processes. Monetary policy in the model is described by the Taylor rule in Equation (3): the policy instrument changes only gradually (with smoothing parameter $\rho_{i}$ ) in reaction to fluctuations in inflation or the output gap, with reaction coefficients given by $\psi_{\pi}$ and $\psi_{x}$. The term $\varepsilon_{t}$ denotes an i.i.d. monetary policy shock.

Expectations $\tilde{E}_{t}$ denote aggregate expectations in the economy, and are obtained as a convex combination of heterogeneous expectation operators. Fractions $n_{x}$ and $n_{\pi}$ of agents are assumed to form conventional rational expectations, denoted here by the mathematical expectation operator $E_{t}$. The remaining fractions $\left(1-n_{x}\right)$ and $\left(1-n_{\pi}\right)$ of agents form expectations that deviate from rational expectations and are denoted by $\hat{E}_{t}$ :

$$
\begin{aligned}
& \tilde{E}_{t} x_{t+1}=n_{x} E_{t} x_{t+1}+\left(1-n_{x}\right) \hat{E}_{t} x_{t+1} \\
& \tilde{E}_{t} \pi_{t+1}=n_{\pi} E_{t} \pi_{t+1}+\left(1-n_{\pi}\right) \hat{E}_{t} \pi_{t+1}
\end{aligned}
$$

Non-rational expectations $\hat{E}_{t}$ are formed as

$$
\begin{aligned}
& \hat{E}_{t} x_{t+1}=\theta_{x}^{2} x_{t-1} \\
& \hat{E}_{t} \pi_{t+1}=\theta_{\pi}^{2} \pi_{t-1} .
\end{aligned}
$$

These expectations can be adaptive, if $0 \leq \theta<1$, naïve, if $\theta=1$, or extrapolative (trend-chasing), when $\theta>1$.

Introducing heterogeneity of expectations into a dynamic general equilibrium may complicate the aggregate dynamics, as aggregation into IS and AS relations is not obvious. Branch and McGough (2009) provide the microfoundations for heterogeneity and discuss the axioms that are needed to ensure that the laws of motions for aggregate variables in the model remain tractable and are easily comparable to the case of rational expectations. Most importantly, restrictions on expectations need to ensure that the law of iterated expectations holds both at the individual and the aggregate level (so that higher-order beliefs don't matter), and that agents have the same expectations about limiting wealth (so that there's no wealth heterogeneity in the long run and no need to track the wealth distribution as a state variable). 


\subsection{Determinacy and Indeterminacy Regions}

In the benchmark, and nested, New Keynesian model, with $n_{x}=n_{\pi}=1$, the determinacy condition

is given by the "Taylor principle": $\psi_{\pi}+\frac{(1-\beta)}{\kappa} \psi_{x}>1$ (Bullard and Mitra, 2002, Woodford, 2003). The aggressiveness of monetary policy toward inflation (and, to a lower extent, toward the output gap) is the main driver of stability in the system. In the New Keynesian model with heterogeneous expectations, however, the state of beliefs has the potential to significantly alter the stability of the equilibrium.

Figures 1 and 2 show the determinacy and indeterminacy regions, obtained by fixing the structural parameters at the prior means shown in Table 1, and by varying the monetary policy and expectation parameters. For ease of exposition, we assume $n_{x}=n_{\pi}$ and $\theta_{x}=\theta_{\pi}$.

When $\theta \leq 1$, the determinacy region expands as the share of adaptive agents increase (the Figure shows the cases for $n_{x}$ and $n_{\pi}$ equal to $0.5,0.8,0.9,0.99$, and 1$)$. On the other hand, when $\theta>1$ indeterminacy becomes much more likely. Even relatively modest shares of non-RE agents are enough to alter the determinacy properties considerably.

Therefore, whether monetary policy is passive or active (by satisfying or not the Taylor principle) may cease to be the main determinant of macroeconomic stability. It's an empirical question whether the existence of heterogeneous expectations changes the conclusions about monetary policy and macroeconomic stability that are typically documented in the literature.

\section{Bayesian Estimation under Determinacy/Indeterminacy}

We estimate both the benchmark New Keynesian model with homogeneous expectations and the extended model with heterogeneous expectations, using a rolling-window approach that allows us to provide evidence on structural parameter instability. For both models, we estimate specifications that accommodate either determinacy or indeterminacy of the equilibrium.

We consider two window sizes. The benchmark results are presented for a rolling window lasting twenty years; in the robustness section, we show the results obtained with a ten-year window. The model is, therefore, re-estimated every year beginning in 1954:Q3 and until 2007:Q3 (we stop before the Great Recession to avoid the effective-lower-bound nonlinearity). We use quarterly time series for output growth, inflation, and the Federal Funds rate as observables. Output growth and inflation are computed as the log first-differences of Real GDP and the GDP Implicit Price Deflator, 
respectively. All variables are obtained from the Federal Reserve Economic Database (FRED).

The model is estimated using Bayesian methods. We use the approach proposed by Bianchi and Nicoló (2019) to estimate the model under indeterminacy. The approach consists of appending to the model equations an auxiliary variable $\omega_{t}$, which follows an autoregressive process:

$$
\omega_{t}=\frac{1}{\alpha} \omega_{t-1}+\zeta_{t}-\eta_{t}^{i}
$$

where $\alpha$ is a positive coefficient, $\zeta_{t}$ is a sunspot shock, and $\eta_{t}^{i}$, with $i=x, \pi$, is one of the expectational errors $^{2}$ (in our model, either related to the output gap or inflation). ${ }^{3}$ When the model is already determinate, the autoregressive coefficient for the auxiliary process is below one $(\alpha>1)$, and the auxiliary variable is redundant, as it doesn't affect any other variable in the system. When the model is indeterminate, the autoregressive coefficient goes above one $(\alpha<1)$ to restore the necessary number of explosive eigenvalues. The auxiliary process $\omega_{t}$ again doesn't influence the remaining parts of the system, but it permits the inclusion of a sunspot shock by inducing a mapping between the expectational errors $\eta_{t}^{i}$ and the sunspot $\zeta_{t} .{ }^{4}$

For each year, we estimate observations in our rolling window, by using a Metropolis-Hastings algorithm to generate draws from the posterior distribution. ${ }^{5}$ Therefore, we estimate 34 (44) windows of observations under the 20-year (10-year) window.

Our prior choices are shown in Table 1. The intertemporal elasticity of substitution $\sigma$ follows a Gamma prior with mean 2 and standard deviation 0.75. The Calvo parameter $\xi_{p}$ follows a Beta distribution with mean 0.7 and standard deviation 0.1 . The monetary policy reaction coefficients for inflation and the output gap follow $N(1.1,0.35)$ and $N(0.25,0.12)$ distributions. We choose a mean just above one, with a sufficiently large standard deviation, for the inflation response, to let the data speak freely on the probability of determinacy versus indeterminacy. All the autoregressive parameters follow Beta distributions to maintain their support between 0 and 1.

For the parameters related to heterogeneous expectations, we assume a Beta prior with mean 0.7 and standard deviation 0.2 for the shares of non-RE agents $n_{x}$ and $n_{\pi}$; these priors slightly favor

\footnotetext{
${ }^{2}$ As shown by Bianchi and Nicoló (2019), the choice of expectational errors to include in (10) does not affect the solution. In this paper, we will make the modelling assumption that the expectational error included in (10) corresponds to inflation.

${ }^{3}$ We write the equation for the case of indeterminacy of order 1 , which is the empirically relevant case here. We have run estimations allowing for indeterminacy of order 2, but those cases are rejected by the data.

${ }^{4}$ Bianchi and Nicoló's methodology is a simplification over previous approaches proposed by Lubik and Schorfheide (2004) and Farmer et al. (2015); in all cases, however, the solutions under indeterminacy remain equivalent.

${ }^{5}$ For each year in the sample, we use 300,000 draws and discard the first $33 \%$ as burn-in.
} 
the benchmark hypothesis of rational expectations, but they are sufficiently diffuse to allow large deviations. ${ }^{6}$ We assume a Gamma prior with mean equal to 1 and standard deviation 0.5 for $\theta_{x}$ and $\theta_{\pi}$ : we center the prior at one, given the importance of this value for determinacy/indeterminacy, and we allow for a large standard deviation, since the parameter may be expected, a priori, to be closer to zero, at least in some parts of the sample.

Finally, we assume inverse Gamma distributions for the fundamental and sunspot shocks; the sunspot is assumed to be potentially correlated with the fundamental shocks. We use a $U(-1,1)$ prior for the correlation coefficients. As discussed in Bianchi and Nicoló (2019), leaving the correlations unrestricted guarantees that the results don't depend on which expectational error (related to inflation or the output gap) is included in the appended equation for the auxiliary variable $\omega_{t}$.

\section{Empirical Results}

\subsection{Homogeneous vs. Heterogeneous Expectations}

Before turning to the analysis of time variation in the model parameters and determinacy of the equilibrium, we provide evidence on the importance of heterogeneous expectations. Figure 3 reports the marginal likelihoods for model specifications with different expectational assumptions over all windows. The model with heterogeneous expectations outperforms the rational, homogeneous, expectations specification decisively and everywhere in the sample.

\subsection{Parameter Variation}

Most DSGE model estimations force the parameters to remain constant over the whole sample of interest. Here, we allow all parameters to vary over time, through our rolling window estimation.

Figures 4, 5, and 6, show the evolution of the estimated posterior means, along with the associated $90 \%$ credibility bands, for three sets of parameters: structural, including policy, parameters, expectation parameters, and parameters related to exogenous shocks.

Monetary policy responds to inflation quite passively in the early samples spanning the years between the 1950s and the mid-1970s, with coefficient between 0.4 and 0.6. The response is slightly lower than 1 later on, and it jumps above one in correspondence of the window starting in 1978 (it reverts back occasionally afterwards). The response to the output gap $\psi_{x}$ is lower in windows that include the 1950s-1960s and 1980s-2000s, but it goes up as a result of the 1970s. The intertemporal

\footnotetext{
${ }^{6}$ We have also experimented with Uniform and $\operatorname{Beta}(0.5,0.25)$ priors with similar results.
} 
elasticity of substitution and the degree of price stickiness increase somewhat over the sample; increases in prices stickiness are consistent with the progressive reduction in the slope of the Phillips curve that has been documented in various studies. The estimates of $\gamma$ capture the productivity slowdown in the 1970s, with posterior means falling below 0.6 , down from the highs around 0.8 in the 1960 s and 1990s.

The paper also provides evidence on time variation regarding the importance of heterogeneous expectations. The shares of RE agents are high over the initial windows (with values around 0.8 or above), but they subsequently fall around 0.2 for output expectations and 0.4 for inflation expectations. The share of non-rational expectations in inflation jumps back to 0.8 in correspondence of the 1978-1998 window. The shares point to evidence of large deviations from rationality: backward-looking expectations are more prevalent than rational expectations for vast periods of time. The parameters related to expectations also drift over the sample. For output, $\theta_{x}$ fluctuates in the neighborhood of 1 for most of the sample. For inflation, expectations are often adaptive, with $\theta_{\pi}<1$, but they become extrapolative for the windows starting in 1977-1978. ${ }^{7}$

As documented in the literature, the standard deviations of the exogenous shocks decrease in the later samples: from 0.6 to 0.3 for demand shocks, from 0.3 to 0.18 for cost-push shocks, and from 0.32 to 0.13 for monetary policy shocks.

\subsection{Heterogeneous Expectations \& Equilibrium Determinacy/Indeterminacy}

In the model with heterogeneous expectations, determinacy of the equilibrium depends on the interaction between monetary policy and expectation formation parameters. As shown in Figures 1 and 2 , when beliefs are characterized by $\theta<1$, larger shares of non-rational agents make determinacy much more likely, and, under some parameter configurations, almost guaranteed. When $\theta>1$, the Taylor principle does not suffice, and the economy may be much more prone to sunspot-driven fluctuations.

We let the data speak about the likelihood of unique versus multiple equilibria. Figure 7 shows the posterior model probability for the model specifications with determinacy versus indeterminacy over the sample. Indeterminacy prevails for estimation windows between 1955-1975 until 1961-1981, with model probabilities reaching up to 1, while the data prefer determinacy starting from samples

\footnotetext{
${ }^{7} \mathrm{~A}$ rise in the perceived persistence of inflation in the $1970 \mathrm{~s}$ is also estimated in models where agents form expectations through adaptive learning, as in Milani (2007, 2011).
} 
beginning in 1962/1963. Despite evidence of quite passive monetary policy in this period (as shown in Figure 4), adaptive behavior in the formation of expectations, especially with inflation expectation coefficients $\theta_{\pi}$ falling below 0.5 and share of non-RE agents above $50 \%$, restores determinacy in the system. The data then reject indeterminacy clearly, until encountering windows starting in 1974, when the probability of indeterminacy rises to 0.3. As seen in Figure 5, expectations show signs of extrapolative behavior in the late 1970s, in a period when monetary policy starts reacting more aggressively toward high inflation (windows that start around Volcker's appointment have Taylor rule coefficients to inflation above one). Extrapolative expectations about output (in 1974) and, more importantly, about inflation (between 1976 and 1979) increase the probability of indeterminate equilibria, which rises to values around 0.5 and 0.7 . In the absence of heterogeneous expectations, indeterminacy would be ruled out in these samples. Finally, post-1983 windows are consistent with determinate equilibria. Even though large shares of non-RE agents populate the economy, their expectations are formed in an adaptive manner, and, therefore, they represent a stabilizing force in the system.

\subsection{Propagation of Shocks}

Passive monetary policy and/or extrapolative expectations may cause the economy to be driven by sunspot shocks. Figure 8 shows the share of output gap and inflation variance that can be explained by sunspots over time. Sunspot-driven fluctuations are important during the early windows, with sunspot shocks accounting for $15-20 \%$ of the variability in output and inflation. They become relevant again in the samples around the late 1970s and very early 1980s, when they are driven by extrapolative expectations in inflation. In the same periods, however, they don't cause large fluctuations in the output gap.

The propagation of fundamental shocks in the economy is also considerably different in the cases of determinacy versus indeterminacy, and under homogeneous versus heterogeneous expectations.

Figure 9 shows the responses of macroeconomic variables to shocks over three different samples: the first is representative of the 1950-1970 samples, which is characterized by passive monetary policy and indeterminacy; the second refers to the later part of the sample (1985-2005), with a stable macroeconomic environment and active policy; the last one relates to the sample starting in 1978, where there's uncertainty about determinacy versus indeterminacy and expectations appear extrapolative. 
In the post-1985 samples, the responses are unsurprising. Monetary policy contractions lead to declines in the output gap and inflation, positive demand shocks generate sluggish increases in inflation and interest rates, and cost-push shocks have small effects on real activity. Sunspots don’t matter as the equilibrium is determinate in this period.

The responses are substantially different in the 1958-1978 sample, chosen to represent the early windows. Under indeterminacy, contractionary monetary policy is unable to contain inflationary pressures: inflation strongly increases in response to positive monetary policy shocks. Demand shocks induce an opposite response of inflation compared with the determinate case. Negative supply shocks induce higher inflation, lower interest rates, and have much larger recessionary effects than in later windows. In addition to fundamental shocks, the economy in the period is under the influence of sunspots: the sunspot $\zeta_{t}$ moves output gap, inflation, and interest rates in the same direction.

Finally, the responses for the 1978-1998 window, which has almost equal probability of determinacy/indeterminacy, and is characterized by trend-chasing expectations, fall typically closer to the case of determinacy, but with some exceptions. In particular, the responses of interest rates to demand and supply shocks are positive and larger than they are under determinacy post-1985. Sunspot shocks matter for inflation, although to a lower extent than in the previous windows, and they are quantitatively unimportant for output.

\section{Robustness}

Our benchmark estimation assumed a conventional timing in the Taylor rule, which allows the central bank to respond to contemporaneous values of inflation and the output gap. A possible alternative consists of assuming that the central bank uses a forecast-based, forward-looking, Taylor rule:

$$
i_{t}=\rho_{i} i_{t-1}+\left(1-\rho_{i}\right)\left(\psi_{\pi} E_{t} \pi_{t+1}+\psi_{x} E_{t} x_{t+1}\right)+\varepsilon_{t}
$$

Responding to forecasts is consistent with central bank parlance, but it is well known to be potentially destabilizing in rational expectations models (Batini and Haldane, 1999). This is no different

for New Keynesian models with heterogeneous expectations. We re-estimate the model with the alternative Taylor rule in the same way using 20-year rolling windows. 
Figure 10 shows how the evidence in favor of determinacy versus indeterminacy, measured by the posterior model probabilities, changes over the sample. We compare the results with those obtained for the benchmark case that was reported in Figure 7. The two cases have similar implications: indeterminacy prevails in the early windows that have their latest observations in the early 1980s. The samples that include Volcker's disinflation, even if they start in the 1960s, are instead consistent with determinacy, as are the post-1984 samples. As expected, the forward-looking Taylor rule raises the probability of indeterminacy, which is now close to one even for windows starting in 1979 to 1983. Again, indeterminacy is then driven by extrapolative behavior in the formation of expectations.

As an additional robustness test, we analyze how the results vary when we shorten the rolling window used in the estimation to ten years. The model probabilities display more variability, given the shorter windows. But the results are overall comparable: indeterminacy prevails for early windows up to those starting in 1973 and ending in 1983, although with various exceptions, suggesting much more pronounced uncertainty with short windows. The subsequent samples are generally consistent with a determinate equilibrium. The main exception is given here by the 19891999 sample, which can be explained equally well as the outcome of determinacy or indeterminacy.

\section{Conclusions}

We estimated a New Keynesian model with heterogeneous expectations to assess the extent of parameter variation and the probability of determinacy and indeterminacy over the sample.

Our empirical results provide substantial evidence in favor of heterogeneous expectations. The model with expectations heterogeneity dominate the benchmark with homogeneous expectations everywhere in the sample. Large shares of agents, at points reaching $80 \%$, who are forming expectations that deviate from the rational expectations hypothesis exist in the economy. The shares of non-RE agents for output and inflation expectations, and the details regarding their expectation process change over the sample. Inflation expectations are typically adaptive, but they become extrapolative for rolling windows starting in the late 1970s, around Volcker's disinflation.

The existence of heterogeneous expectations and the degree of adaptive and extrapolative behavior may modify determinacy conditions in the New Keynesian model and, therefore, may overturn well-known results in the literature. When taken to the data, the model with heterogeneous expectations suggests indeterminacy as a feature that is needed to explain observations in the early 
windows, while determinacy dominates the post-1984 samples. But, under heterogeneous expectations, we uncover new periods that are consistent with large probabilities of indeterminacy, such as samples that begin in the late 1970s, when inflation expectations turn extrapolative.

In light of the favorable empirical evidence for heterogeneous expectations, both at the micro, experimental, and macro level, future research should further investigate the implications of heterogeneity in a wide variety of DGSE models.

\section{References}

[1] Batini, N., and A.G. Haldane (1999). "Forward-looking rules for monetary policy", Bank of England Working Paper No. 91.

[2] Benhabib, J., and R.E.A. Farmer (1999). "Indeterminacy and sunspots in macroeconomics", ch. 06, p. 387-448 in Taylor, J.B. and Woodford, M. eds., Handbook of Macroeconomics, vol. 1, Part A, Elsevier.

[3] Beqiraj, E., Di Bartolomeo, G., Di Pietro, M., and C. Serpieri (2018). "Bounded-rationality and heterogeneous agents: long or short forecasters?", JRC Working Papers JRC111392, Joint Research Centre.

[4] Beqiraj, E., Di Bartolomeo, G., and C. Serpieri (2019). "Rational vs. long-run forecasters: optimal monetary policy and the role of inequality, Macroeconomic Dynamics, 23(S1), pages $9-24$.

[5] Bianchi, F., and G. Nicoló (2019). "A Generalized Approach to Indeterminacy in Linear Rational Expectations Models", Finance and Economics Discussion Series 2019-033, Board of Governors of the Federal Reserve System.

[6] Branch, W.A. (2004), "The theory of rationally heterogeneous expectations: Evidence from survey data on inflation expectations, The Economic Journal, 114(497): 592-621.

[7] Branch, W.A. (2014). "Nowcasting and the Taylor rule", Journal of Money, Credit and Banking, 46(5), 1035-1055.

[8] Branch, W.A., and B. McGough (2009). "A New Keynesian model with heterogeneous expectations, Journal of Economic Dynamics and Control, 33(5), 1036-1051.

[9] Branch, W.A. and B. McGough (2011). "Business cycle amplification with heterogeneous expectations", Economic Theory, 47(2), 395-421.

[10] Branch, W.A., and B. McGough (2018). "Heterogeneous expectations and micro-foundations in macroeconomics, Handbook of Computational Economics, K. Schmedders and K.L. Judd (eds.), Elsevier Science, North-Holland, Vol. 4. 
[11] Bullard, J.B., and K. Mitra (2002). "Learning about monetary policy rules, Journal of Monetary Economics, 49, 1105-1129.

[12] Calvert Jump, R., and P. Levine (2019). "Behavioural New Keynesian models", Journal of Macroeconomics, 59, 59-77.

[13] Canova, F. (2009). "What explains the Great Moderation in the U.S.? A structural analysis", Journal of the European Economic Association, 7: 697-721.

[14] Canova, F., and F. Ferroni (2012). "The dynamics of US inflation: Can monetary policy explain the changes?", Journal of Econometrics, 167(1), 47-60.

[15] Castelnuovo, E. (2012). "Fitting U.S. trend inflation: a rolling-window approach", Advances in Econometrics, Vol. 28, pp. 201-252.

[16] Clarida, R., Galí, J., and M. Gertler (2000). "Monetary policy rules and macroeconomic stability: evidence and some theory", The Quarterly Journal of Economics, 115(1), 147-180.

[17] Cole, S., and F. Milani (2019). "The misspecification of expectations in New Keynesian models: a DSGE-VAR approach", Macroeconomic Dynamics, 23(3), 974-1007.

[18] Cole, S., and F. Milani (2020). "Heterogeneity in individual expectations, sentiment, and constant-gain learning", mimeo, UC Irvine.

[19] Di Bartolomeo, G., Di Pietro, M., and B. Giannini (2016). "Optimal monetary policy in a New Keynesian model with heterogeneous expectations, Journal of Economic Dynamics and Control, 73: 373-387.

[20] Dovern, J., Fritsche, U., and J. Slacalek (2012). "Disagreement among forecasters in G7 countries", Review of Economics and Statistics, 94(4), 1081-1096.

[21] Farmer, R.E.A., Khramov, V., and G. Nicoló (2015). "Solving and estimating indeterminate DSGE models", Journal of Economic Dynamics and Control, 54, 17-36.

[22] Fernández-Villaverde, J., and J.F. Rubio-Ramírez (2007). "How structural are structural parameters?," NBER Chapters, in: NBER Macroeconomics Annual, Volume 22, pages 83-137, National Bureau of Economic Research, Inc.

[23] Gasteiger, E. (2014). "Heterogeneous expectations, optimal monetary policy, and the merit of policy inertia", Journal of Money, Credit and Banking, 46(7), 1533-1554.

[24] Gasteiger, E. (2018). "Do heterogeneous expectations constitute a challenge for policy interaction?", Macroeconomic Dynamics, 22(8), 2107-2140.

[25] Hommes, C.H. (2011). "The heterogeneous expectations hypothesis: Some evidence from the lab", Journal of Economic Dynamics and Control 35(1), 1-24. 
[26] Hommes, C.H. (2013). "Behavioral rationality and heterogeneous expectations in complex economic systems", Cambridge University Press.

[27] Ilabaca, F., Meggiorini, G., and F. Milani (2020). "Bounded rationality, monetary policy, and macroeconomic stability", Economics Letters, Volume 186, Article 108522.

[28] Lubik, T.A. and F. Schorfheide (2004). "Testing for indeterminacy: an application to U.S. monetary policy", American Economic Review, 94, 190-219.

[29] Mankiw, N.G., Reis, R., and J. Wolfers (2004). "Disagreement about inflation expectations", NBER Chapters, in: NBER Macroeconomics Annual, Volume 18, pages 209-270, National Bureau of Economic Research, Inc.

[30] Massaro, D. (2013). "Heterogeneous expectations in monetary DSGE models", Journal of Economic Dynamics and Control, 37, 680-692.

[31] Milani, F. (2007). Expectations, learning and macroeconomic persistence", Journal of Monetary Economics, 54(7), 2065-2082.

[32] Milani, F. (2008). "Learning, monetary policy rules, and macroeconomic stability", Journal of Economic Dynamics and Control, 32(10), 3148-3165.

[33] Milani, F. (2011). "Expectation shocks and learning as drivers of the business cycle", Economic Journal, 121(552), 379-401.

[34] Pesaran, M.H., and M. Weale, (2006). "Survey expectations", Handbook of Economic Forecasting, in: G. Elliott C. Granger A. Timmermann (ed.), Handbook of Economic Forecasting, edition 1, volume 1, chapter 14, pages 715-776, Elsevier.

[35] Woodford, M. (2003). Interest and Prices: Foundations of a Theory of Monetary Policy. Princeton, NJ: Princeton University Press. 


\begin{tabular}{ll|ll}
\hline \hline & \multicolumn{2}{c}{ Prior Distributions } & \\
\hline IES & $\sigma \sim \Gamma(2,0.75)$ & AR Demand & $\rho_{g} \sim B(0.5,0.2)$ \\
Calvo price stick. & $\xi_{p} \sim B(0.7,0.1)$ & AR Cost-Push & $\rho_{u} \sim B(0.5,0.2)$ \\
Int. Rate Smooth. & $\rho_{i} \sim B(0.7,0.2)$ & Std MP shock & $\sigma_{\varepsilon} \sim I G(0.5,2)$ \\
MP Inf & $\psi_{\pi} \sim N(1.1,0.35)$ & Std Demand shock & $\sigma_{g} \sim I G(0.5,2)$ \\
MP Output Gap & $\psi_{x} \sim N(0.25,0.12)$ & Std Cost-Push shock & $\sigma_{u} \sim I G(0.5,2)$ \\
Share non-RE in Output & $n_{x} \sim B(0.7,0.2)$ & Std Sunspot shock & $\sigma_{\zeta} \sim I G(0.5,2)$ \\
Param. Output Exp & $\theta_{x} \sim \Gamma(1,0.5)$ & Corr Sunspot \& MP shock & $\sigma_{\zeta, \varepsilon} \sim U(-1,1)$ \\
Share non-RE in Infl & $n_{\pi} \sim B(0.7,0.2)$ & Corr Sunspot \& Dem shock & $\sigma_{\zeta, \varepsilon^{g}} \sim U(-1,1)$ \\
Param. Infl Exp & $\theta_{\pi} \sim \Gamma(1,0.5)$ & Corr Sunspot \& CP shock & $\sigma_{\zeta, \varepsilon^{u}} \sim U(-1,1)$ \\
Output growth & $\gamma \sim N(0.675,0.15)$ & & \\
S.S. infl & $\bar{\pi} \sim \Gamma(0.75,0.4)$ & & \\
S.S. real rate & $\bar{r} \sim \Gamma(0.5,0.35)$ & & \\
\hline \hline
\end{tabular}

Table 1: Prior Distributions for estimated coefficients.

Note: $\Gamma$ refers to Gamma distribution, $B$ to Beta, $N$ to Normal, $I G$ to Inverse Gamma, and $U$ to Uniform. The numbers in parenthesis refer to mean and standard deviations, with the exception of Uniform priors, for which we report the lower and upper bounds. 


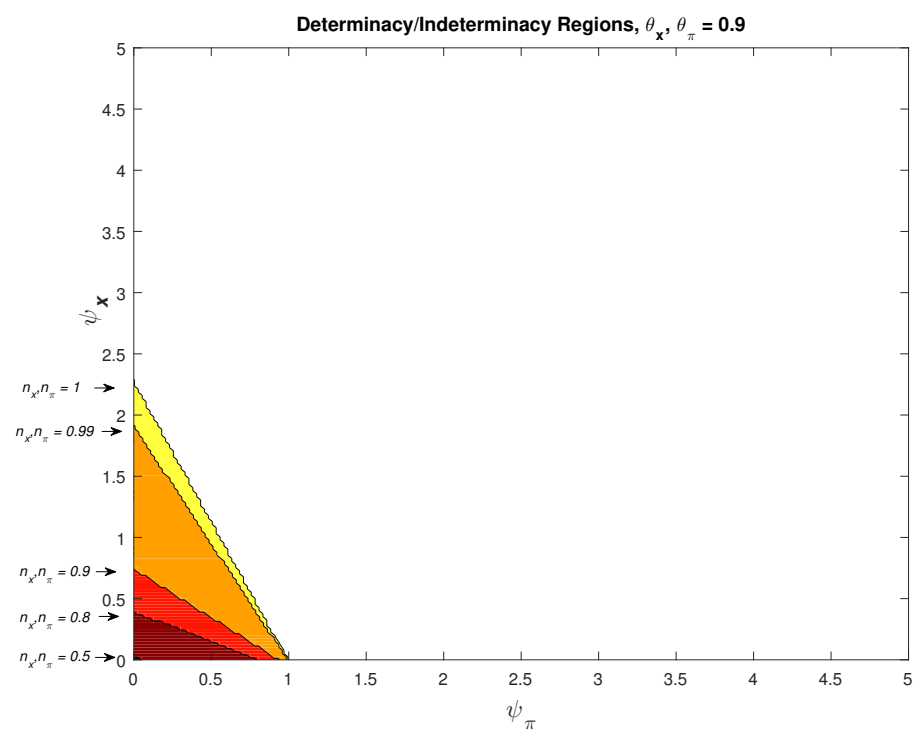

Figure 1: Determinacy and Indeterminacy Regions: case with $\theta_{\pi}=\theta_{x}=0.9$.

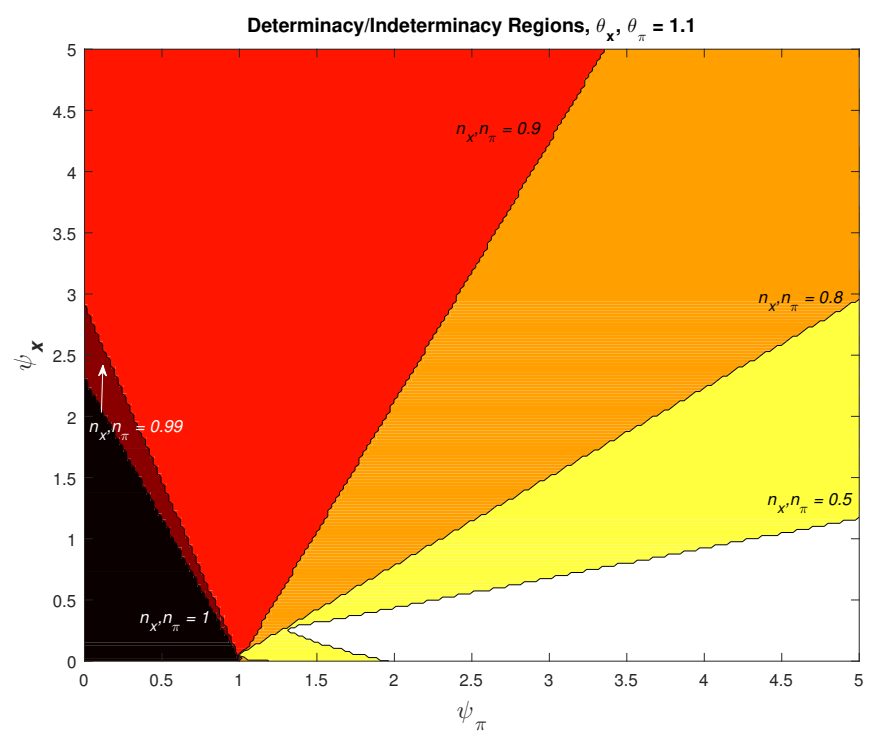

Figure 2: Determinacy and Indeterminacy Regions: case with $\theta_{\pi}=\theta_{x}=1.1$. 


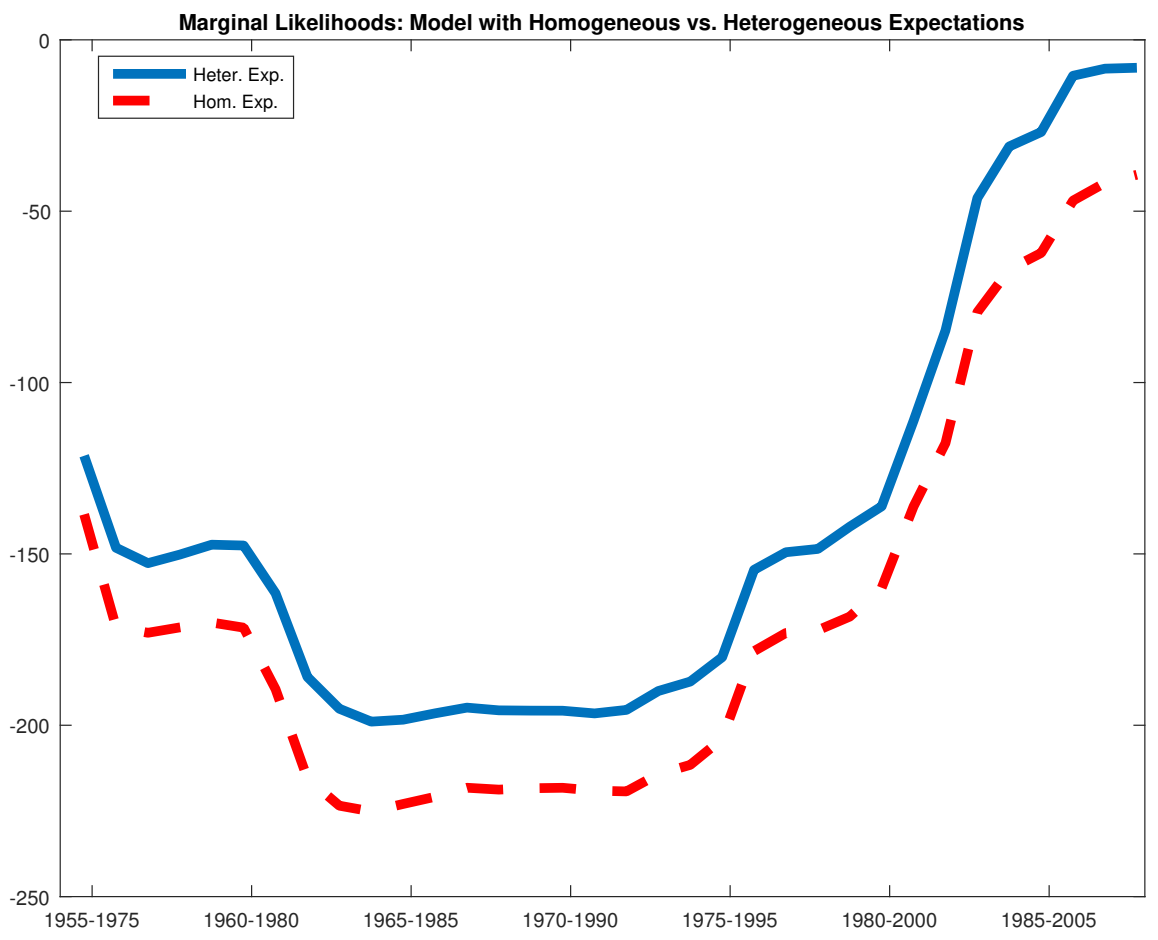

Figure 3: Bayesian Model Comparison: marginal likelihoods for the New Keynesian model under homogeneous (red, dashed) versus heterogeneous (blue, solid) expectations over time. 

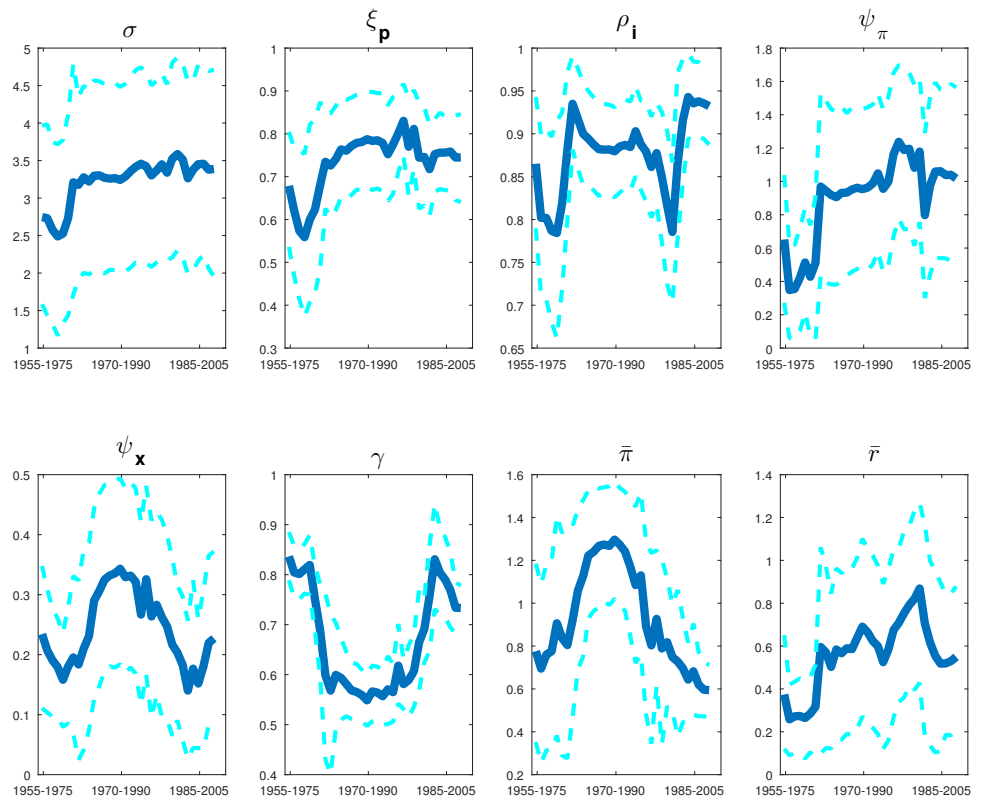

Figure 4: Parameter variation over the sample: 20-year rolling window estimation, 'structural' parameters.
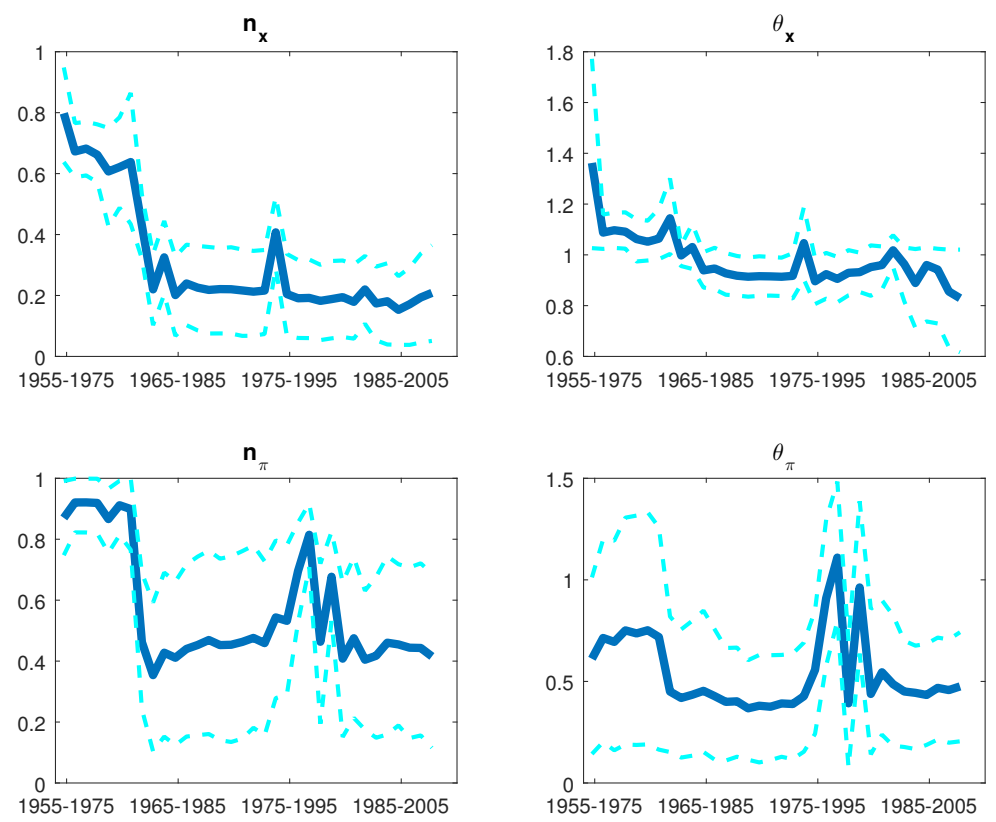

Figure 5: Parameter variation over the sample: 20-year rolling window estimation, expectation parameters. 

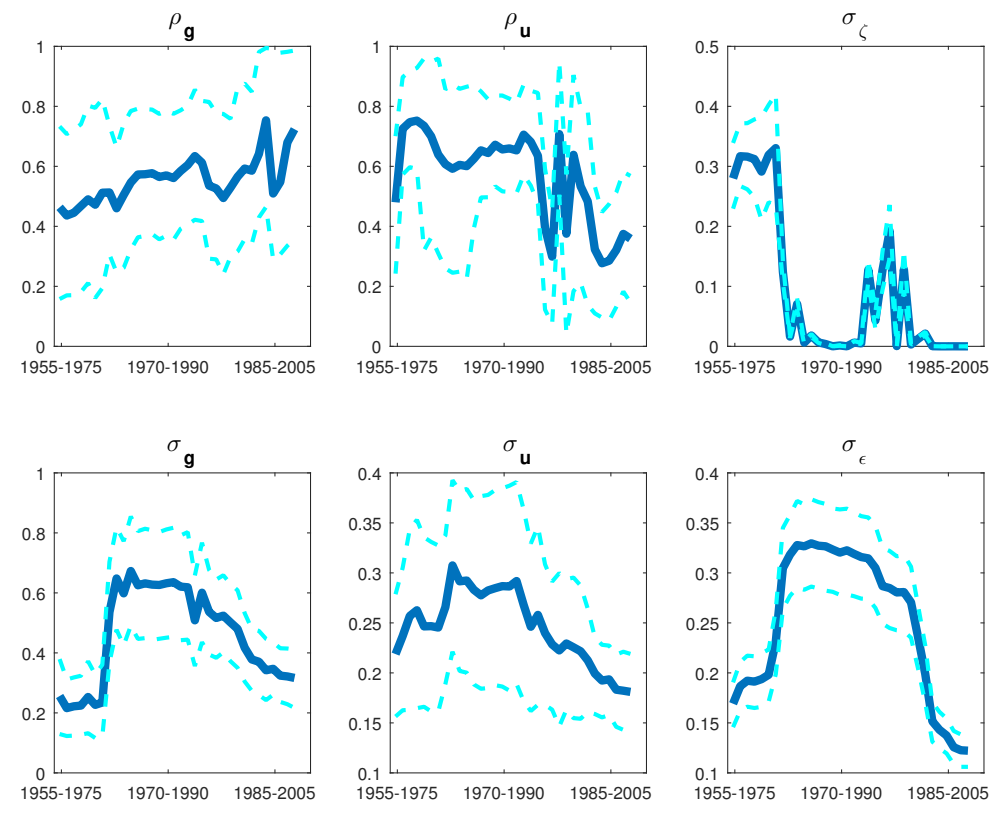

Figure 6: Parameter variation over the sample: 20-year rolling window estimation, disturbance parameters. 


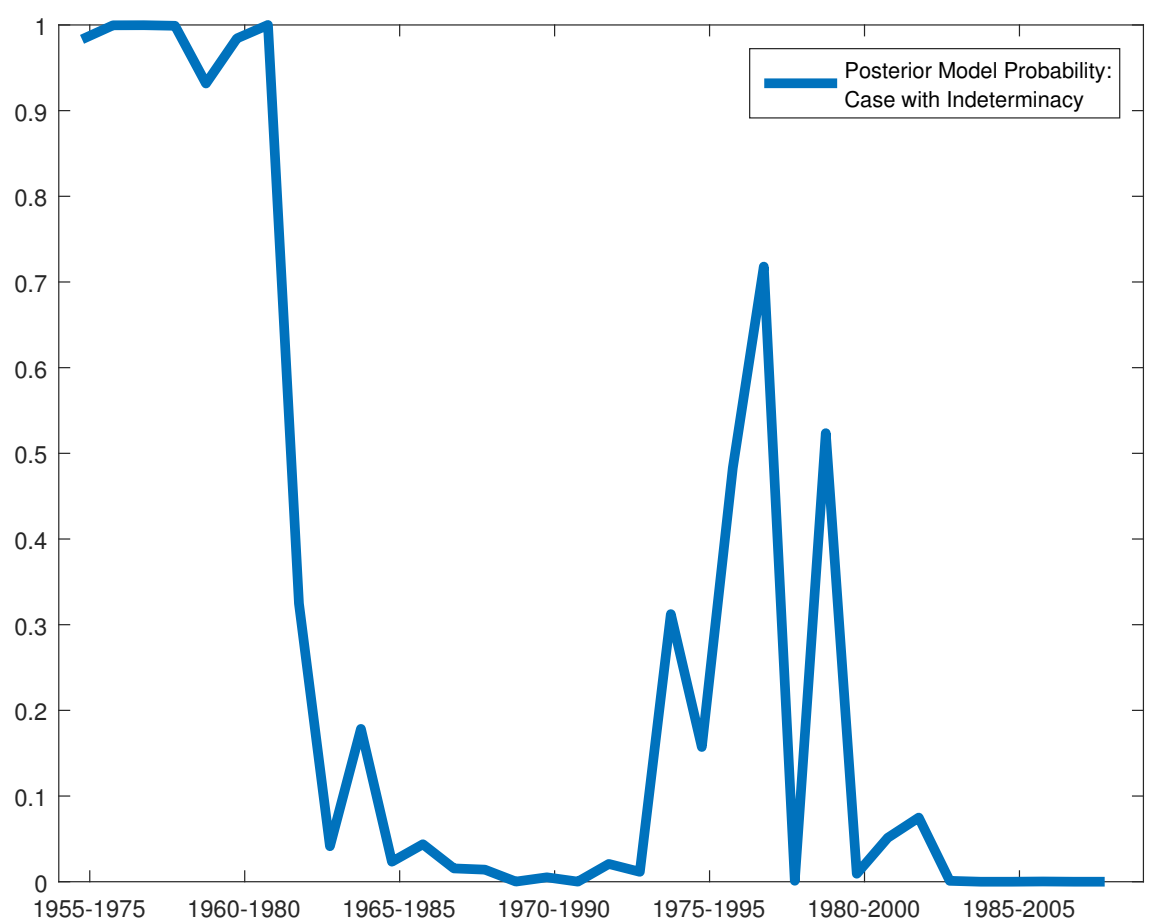

Figure 7: Posterior Model Probabilities over time: Specification with Indeterminacy versus Determinacy. 

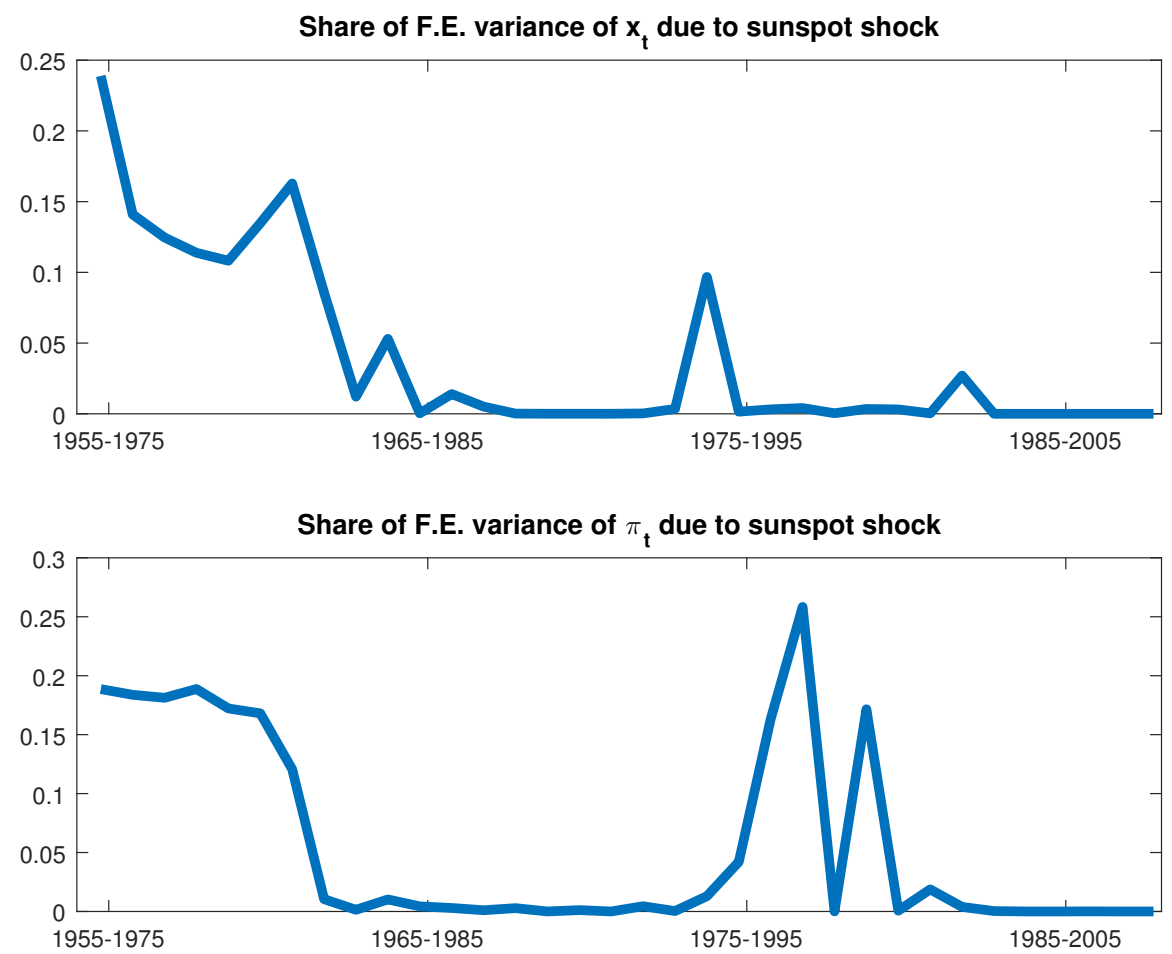

Figure 8: Forecast Error Variance Decomposition: share of variance in output gap (top panel) and inflation (bottom panel) explained by sunspot shock, over the sample. 

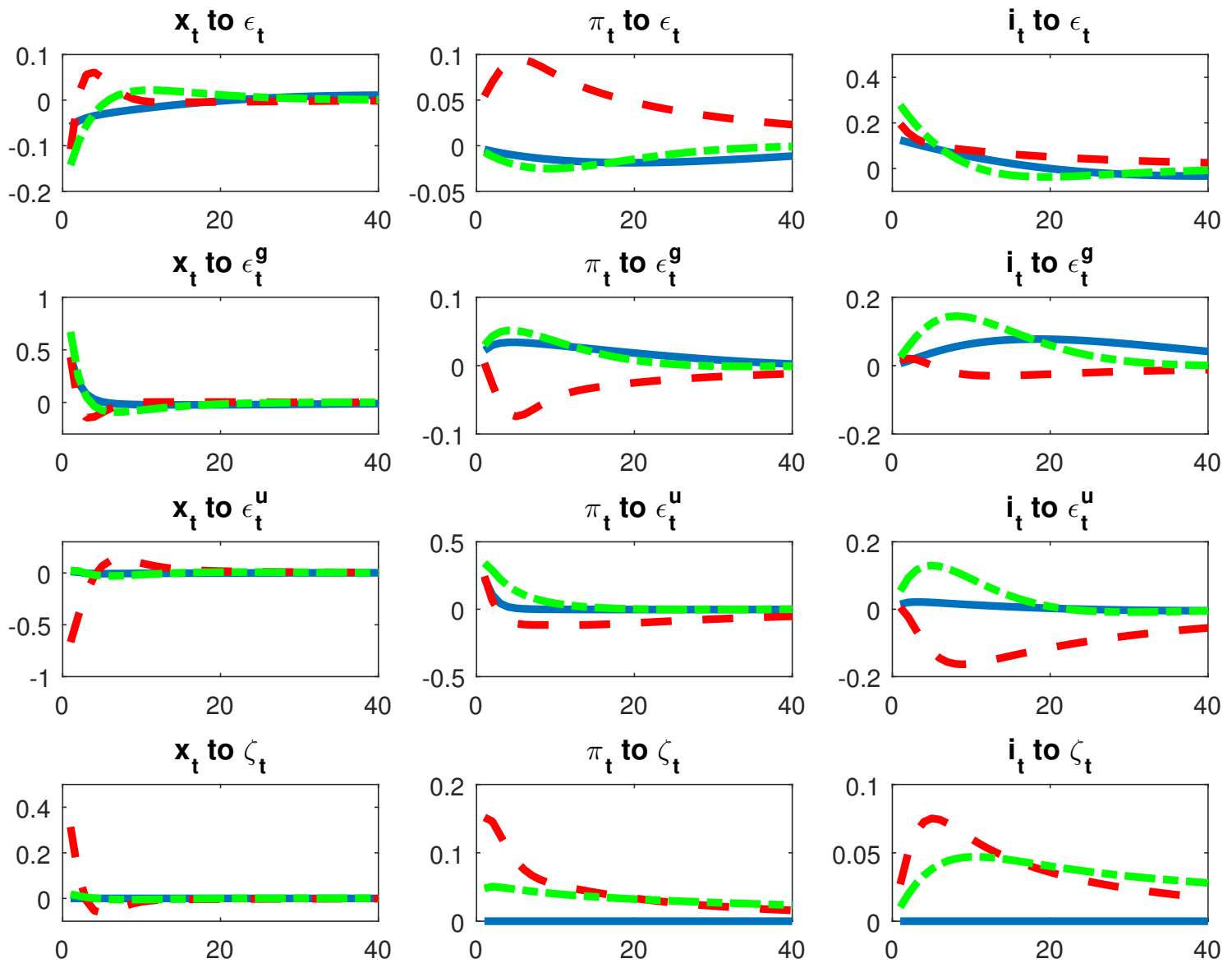

Figure 9: Propagation of Fundamental and Sunspot Shocks in different samples.

Note: The figure shows the response of the output gap, inflation, and interest rates, to monetary policy shocks (first row), demand shocks (second row), cost-push shocks (third row), and sunspot shocks (fourth row). Impulse responses are means across Metropolis-Hastings draws. The solid blue line refers to the 1985-2005 window, the dash-dot green line to the 1978-1998 window, and the dashed red line to the 1958-1978 window. 

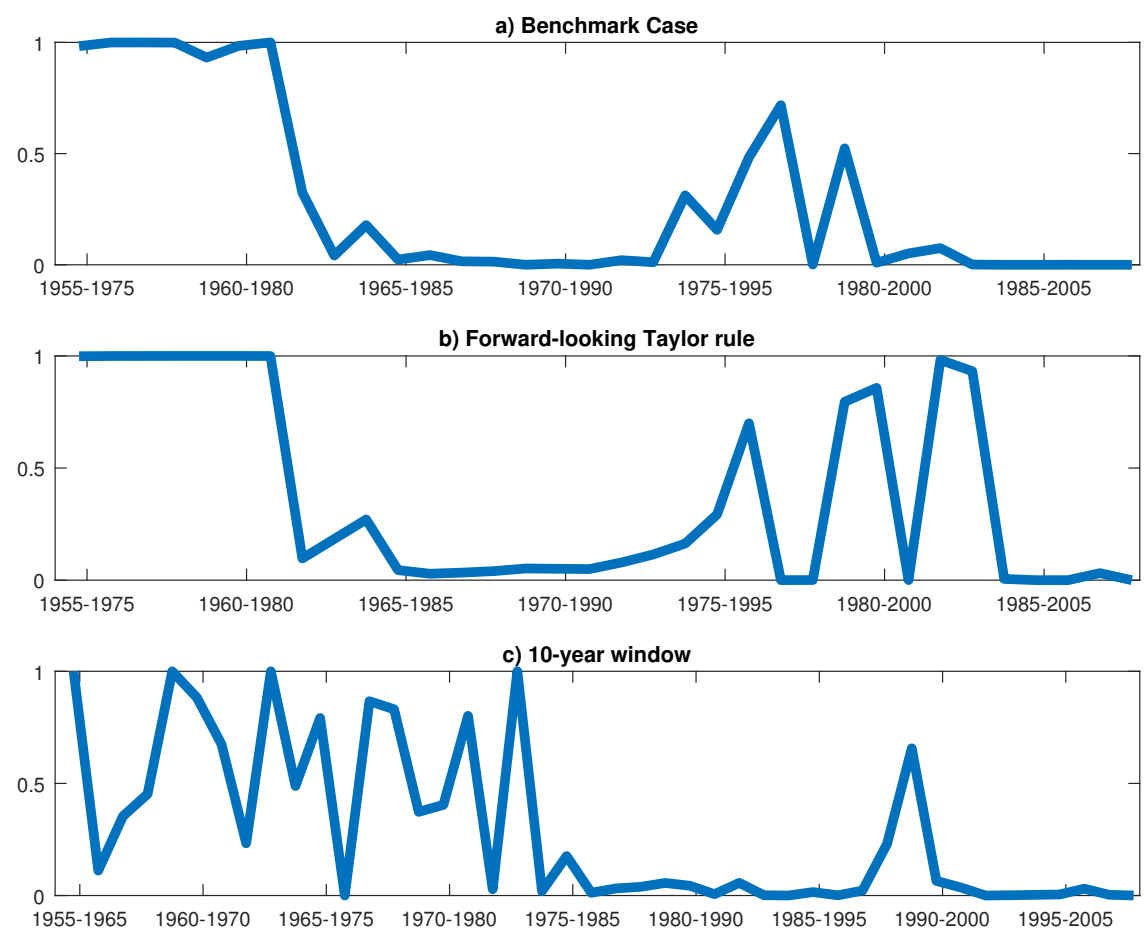

Figure 10: Robustness analysis: posterior model probabilities in favor of indeterminacy versus determinacy.

Notes: The figure shows the posterior model probabilities for three cases: a) the same benchmark case presented in Figure 7 (added for comparison) with 20-year rolling windows; b) the case with a forward-looking Taylor rule; c) the case with a 10-year rolling window. 